\title{
Correlation between wall temperature and flow field of an impinging chevron jet
}

\author{
by M. Contino*, C. S. Greco*, T. Astarita* and G. Cardone* \\ *University of Naples Federico II, Department of Industrial Engineering, Via Claudio 21, 80125, Naples, Italy, \\ mattia.contino@unina.it, carlosalvatore.greco@unina.it, tommaso.astarita@unina.it, gennaro.cardone@unina.it
}

\begin{abstract}
InfraRed thermography and Time Resolved Tomographic Particle Image Velocimetry techniques are simultaneously applied to investigate the correlation between heat transport and momentum of a circular and a chevron continuous jet impinging on a flat plate in water. The two different jets configurations are investigated at fixed Reynolds number and nozzle-to-plate distance. Mean and turbulent fluctuation distributions of temperature at impinging surface and of velocity close to the wall are presented overlapped on the same frame. The simultaneous measurements revealed that the local enhancement of the mixing happens along the apices of the chevron nozzle which are characterised by larger velocity fluctuations.
\end{abstract}

\section{Introduction}

The heat transfer topic attracts the scientific research from multiple points of view, one important subject is the investigation of impinging jets [1,2]. This kind of flows gained large attention because of their wide range of applicability (drying, cooling, tempering) and great variety of possible nozzle geometries [3]. In particular, cooling efficiency strictly depends on how much the impacting flow is able to generate a turbulent flow in proximity of the wall, uniformity of cooling flow distribution over the wall and degree of mixing between the fluid which stands over the hot wall and the cooling fluid [4]. In particular, it is of main interest to deeply understand the unsteady behaviour of the flow and the thermal fluctuations in proximity of any critical points like in case of separation or reattachment. Small temperature differences do not govern fluid motion. Hence such kind of contaminant has no dynamical effect on the fluid motion itself so it can be used as additional tracer in the flow under investigation [5].

The most promising non-invasive techniques which let investigate unsteady complex flows from thermal and velocity points of view are respectively the High Speed InfraRed (IR) thermography and Time Resolved Tomographic Particle Image Velocimetry (TR Tomo-PIV). The first technique detects the electromagnetic energy radiated by an object and converts it into a sequence of instantaneous 2D temperature maps [6]. The High Speed IR thermography technique appears advantageous from several points of view. As a matter, an IR camera offer a fully 2D non-intrusive high sensitive (up to $20 \mathrm{mK}$ ) and low response time (order of $\mu \mathrm{s}$ ) measurement of the accessible field of view. The second mentioned technique has been largely employed to provide three-dimensional time-resolved (4D measurements) data offering advanced flow diagnostic possibilities. In particular, a volume-based measurement technique brings the advantage to measure the velocity vector and the possibility to evaluate the vorticity vector [7]. As a consequence, the organization of turbulent coherent structure can be investigated with less ambiguity. Hence both techniques are able to measure the fluctuations of the flow field from the thermal and fluid dynamic points of view with almost the same frame rate without influencing it in any way.

Turbulent flows are typically three-dimensional, unsteady and characterized by a wide range of length and time scales [8]. Interaction between a jet impinging on a wall or mixing of two non-isothermal fluids are usually associated with turbulent phenomena. In particular, the ability to control a flow by manipulating its structure, is one of the most important topics in fluid dynamics. All the control strategies have the common purpose to modulate flow characteristics depending on the goal to reach in each specific application. The control can be achieved through different technical solutions but the most attractive is always the geometrical modification of the nozzle geometry [9]. Such customization requires only an initial investment, but if the performance results are promising, it could be worth starting the large scale production without any additional costs. A specific nozzle geometry dubbed as "super mixer" by NASA is the chevron nozzle [10]. The effects of tabs applied to exhausting nozzle has been extensively studied to reduce noise in supersonic applications without affecting trust [11]. Subsonic applications of chevron exit have been investigated to answer whether such a nozzle geometry could produce significant enhancement to heat transfer capabilities and mixing properties of standard circular jet $[12,13]$. 
In order to achieve a quantitative estimation of the correlation between the velocity and thermal fluctuations, the two measuring techniques have to respectively investigate on two accurately overlapped domains. The IR thermography is a $2 \mathrm{D}$ technique and even a thin layer of liquid water absorbs almost all IR-radiation. Hence, the timeevolving temperature map can be only achieved through the application of an IR transparent window. On the other hand, the TR Tomo-PIV can measure velocity fluctuations close to the wall depending on the particle density, interrogation windows size and overlap distance between adjacent interrogation windows. Through the simultaneous application of the two mentioned techniques the present paper will describe the correlation between near-wall 3D velocity flow field and the thermal footprint of a circular and a six-chevron cold jet impinging from above on a flat horizontal plate placed in a tank filled with hot water.

\section{Experimental apparatus and procedure}

The experimental apparatus is schematically represented in figure 1. The water facility includes two tanks of different capacities: a small one which is filled with cold water (293K) and a bigger one filled with hot water (298K). Both tanks are controlled in temperature with accuracy of $0.03 \mathrm{~K}$. A nozzle $(\mathrm{N})$ (circular or chevron) of diameter $\mathrm{D}=10 \mathrm{~mm}$ is suspended at a fixed distance from wall $(H / D=2)$ over a circular sapphire window (S) (diameter $150 \mathrm{~mm}$, thickness $4 \mathrm{~mm}$ ) sealed on the bottom of the hot tank. On the other side of the window are positioned 4 high speed PIV cameras $\left(C_{i}\right)$ and a Mid-Wavelength InfraRed (MWIR) camera $\left(T_{1}\right)$ which share a common trigger. The test starts when the geared pump $(P)$ pushes the cold water through a flow meter $(F)$. The fluid finally exhausts through the nozzle at a constant mass rate equal to $240 \mathrm{l} / \mathrm{h}$ resulting in a Reynolds number equal to 8100 . After 120 seconds the pump is working, the simultaneous Tomo-Thermographic acquisition starts.

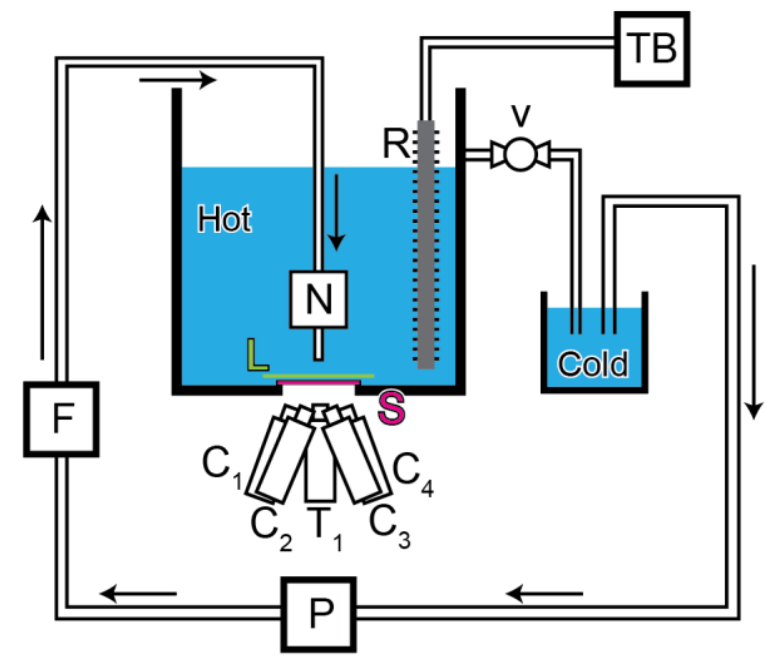

Fig. 1. Scheme of the experimental apparatus. P) Gear type pump; F) Flowmeter; N) Nozzle (circular or chevron); L) Laser volume; S) Sapphire window; TB) Thermal Bath; R) Radiator; V) Valve; $C_{i}$ ) High Speed PIV cameras; $\left.T_{1}\right)$ High Speed IR camera.

The temperature difference is monitored by two RTDs Pt100 (1/10 DIN) placed in the two tanks. Initially the valve $(\mathrm{V})$ is opened and the thermal bath cools down the entire loop through a radiator placed in the larger tank. Once the lower temperature is reached, the pump is stopped and the valve is closed. Then the fluid in the larger tanks is warmed up changing the temperature target of the thermal bath. The choice to manage with such small temperature difference of about $\Delta T=5 \mathrm{~K}$ is justified by the nonlinear behaviour of water density depending on its temperature. With such described conditions, the water will change its density only of $0.1 \%$ passing through the lower temperature to the higher one.

The round nozzle of exit diameter $D=10 \mathrm{~mm}$ and contraction ratio of 56.25 has been characterized in [14]. A sixchevrons exit is applied on top of the circular nozzle profile, with tabs inclined towards the jet axis. The chevron length $\ell$ is $0.43 \mathrm{D}$ and the penetration depth $p$ is $0.134 \mathrm{D}$, according to the model SMC006 used by [10]. A schematic of the chevron nozzle is shown in figure 2.

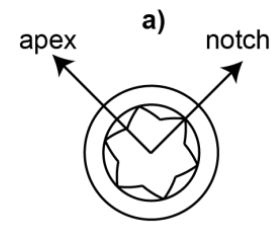

b)

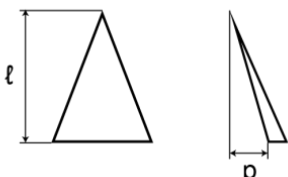

Fig. 2. a) Azimuthal position of chevron notch and apex; b) details of the chevron nozzle: length $\ell$ and penetration depth $p$. 
The developed set up is based on the property of water to absorb radiation at sub-millimetre scale while it is perfectly transparent in the laser wavelength range (figure 3). Hence, this makes possible to measure non-isothermal instantaneous turbulent fluctuations of the fluid in the boundary layer through an IR-transparent window. The most appropriate material for water submerged applications is sapphire. Sapphire optical windows are useful for UV, visible, and IR wavelengths, with transmission from less than $200 \mathrm{~nm}$ out beyond $5 \mu \mathrm{m}$. In addition, Anti-Reflective coating is applied on one side of the window. In particular the coating has close to zero reflectance for wavelength included in the $\mathrm{Nd}$ :YLF laser range (around $527 \mathrm{~nm}$ ) and in the MWIR range $(3-5 \mu \mathrm{m})$ (figure 4).

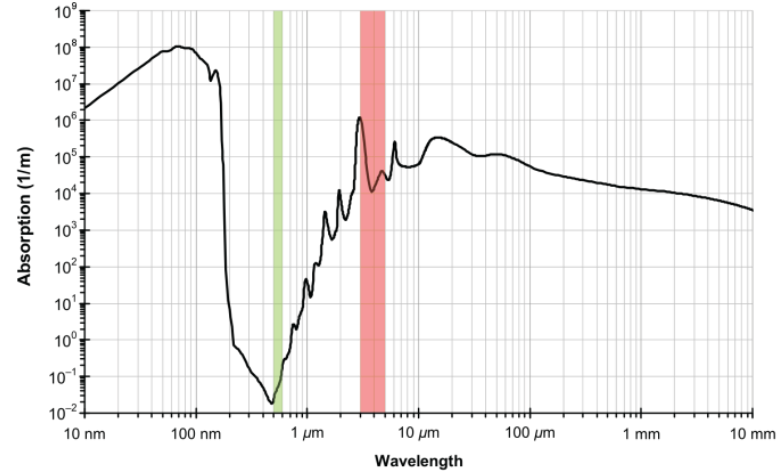

Fig. 3. Absorption spectrum of liquid water adapted from [15]. Nd:YLF laser wavelength range highlighted in green, MWIR range highlighted in red.

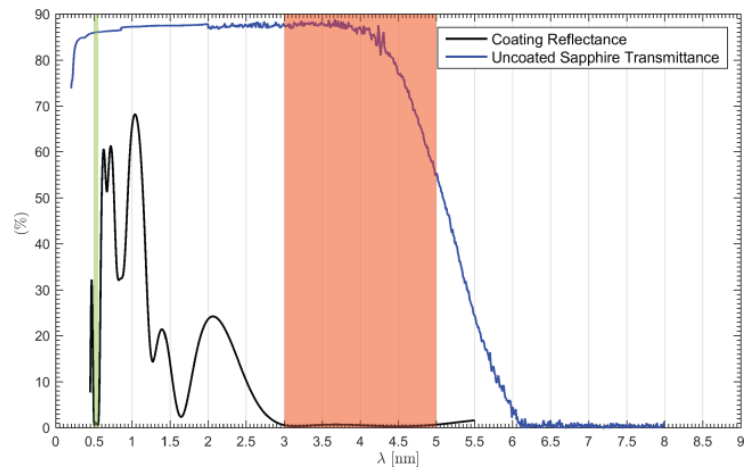

Fig. 4. (black solid line) Reflectance of AntiReflective coating of the sapphire window; (blue solid line) Transmittance of the uncoated sapphire window (courtesy of [16]). Nd:YLF laser wavelength range highlighted in green, MWIR range highlighted in red.

\subsection{InfraRed Thermography}

A CEDIP JADE III (MW) IR camera working in the $3-5 \mu \mathrm{m}$ band is employed to measure the temperature fluctuations through the sapphire window with a spatial resolution of 2.16 pixels $/ \mathrm{mm}$. The camera sensor has nominal dimensions of $320(\mathrm{H}) \times 240(\mathrm{~V})$, pixel pitch of $30 \mu \mathrm{m}$, temperature sensitivity of $0.025 \mathrm{~K}$. For the current application the sensor is used as cropped to $160(\mathrm{H}) \times 120(\mathrm{~V})$ pixels to achieve higher frequency of acquisition. The resulting field of view is 7.3Dx5.5D. Images are acquired at frequency of $400 \mathrm{~Hz}$ and Integration Time (IT) equal to $1100 \mu \mathrm{s}$. A total of $34^{\prime} 000$ samples are acquired starting from the trigger resulting in a time of acquisition of $85 \mathrm{~s}$.

A thermal calibration procedure is needed to correctly remove the background image depending on camera temperature. Background images are acquired for two water temperature (293K and 298K) and for seven camera temperatures (from $305 \mathrm{~K}$ to $311 \mathrm{~K}$, proceeding with $1 \mathrm{~K}$ steps). Through by the application of a linear regression procedure over the seven points associated to each pixel of the thermal image, two interpolated background images at the test camera temperatures are estimated. A linear correction, see Eq. (1), in the selected temperature range provides the instantaneous temperature maps. In Eq. (1) $T_{C}$ and $T_{H}$ are the background images respectively at lower and higher temperature in the selected range, $\Delta T$ is temperature difference between the boundaries of the selected range of temperature, $T_{\text {corr }}$ is the resulting temperature map from the linear correction.

$$
T_{c o r r}=\frac{T-T_{C}}{T_{H}-T_{C}} \cdot \Delta T+T c
$$

\subsection{Time-Resolved Tomo-PIV}

Neutrally buoyant LaVision Polyamide High Quality particles of $56 \mu \mathrm{m}$ of diameter are employed to uniformly seed the water. The illumination is provided by a solid-state diode-pumped LDY303 Nd:YLF laser $(2 \times 20 \mathrm{~mJ})$. The laser beam diameter of $0.5 \mathrm{D}$ is first expanded along the nozzle to wall direction. Then the beam is expanded on the other direction with a cylindrical lens up to $8 \mathrm{D}$ of width. The light scattered by the particles is recorded by a tomographic

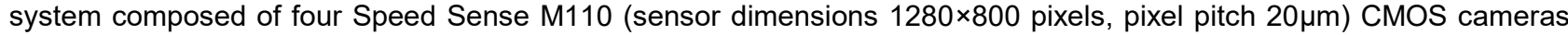
arranged vertically below the hot tank in a cross-like configuration. Optics are mounted on cameras through by 3Dprinted Scheimpflug. Tokina AT-X M100 PRO D objectives of $100 \mathrm{~mm}$ focal length are set with a numerical aperture $\mathrm{f}_{\#}=32$ to allow focused imaging of the illuminated particles. The resulting field of view is $5.1 \mathrm{D} \times 3 \mathrm{D}$ with a digital resolution of $21.5 \mathrm{pixels} / \mathrm{mm}$. For the chosen illumination and imaging configuration the maximum particle image density is approximately 0.03 particles/pixel. Sequences of images of tracer particles are recorded at $1600 \mathrm{~Hz}$. A total of 1700 images are acquired for a total time of acquisition of $1.0625 \mathrm{~s}$.

A three-dimensional mapping function from image-space to physical object-space is generated by imaging a calibration target. The initial experimental errors due to system calibration are estimated at approximately 0.5 pixels by the disparity vector field. The misalignment is reduced to less than 0.05 pixels making use of the self-calibration 
technique [17]. The raw images are pre-processed with subtraction of the minimum intensity at each pixel for the entire sequence, followed by subtraction of the local minimum over a kernel of $7 \times 7$ pixels and application of a Gaussian filter with $\sigma=0.5$. The use of 4-camera tomographic system enables $3 \mathrm{D}$ object reconstructions with a reconstruction quality $Q$ above 0.85 [18]. The volumetric light intensity reconstruction is performed following multiple iterations of diffusive Camera Simultaneous Multiplicative Algebraic Reconstruction Technique algorithm (C-SMART) and diffusive SMART to generate a velocity predictor through a time-marching Sequential Motion Tracking Enhancement (SMTE) reconstruction algorithm. Errors introduced by the predictor are corrected by the "Shake-the-Box" method combined with the image matching introduced by Iterative Particle Reconstruction $[19,20]$. The volume under investigation of approximately $2.5 \mathrm{D} \times 4.5 \mathrm{D} \times 0.8 \mathrm{D}$ is discretized with $535 \times 960 \times 190$ voxels applying a pixel to voxel ratio of 1 . The three-dimensional particle field motion is computed by Volume Deformation Iterative Multigrid technique $[21,22]$ with an interrogation box size decreasing from $96 \times 96 \times 96$ to a final box size of $64 \times 64 \times 64$ voxels, including on average, 12 particles. The size of the final interrogation boxes enables a spatial resolution of $0.297 \mathrm{D} \times 0.297 \mathrm{D} \times 0.297 \mathrm{D}$. In order to increase of the spatial sampling, an overlap factor of $75 \%$ between adjacent interrogation boxes is chosen, leading to a vector pitch of $0.074 \mathrm{D}$. It results that the velocity field is discretized over a grid of $39 \times 67 \times 11$ vectors. To keep high the measurement precision, almost two vectors from each side of the volume has to be neglected. The schematic representation of the Tomographic measurement domain positioning is illustrated in figure 5.

Since the velocity and the thermographic resolutions are much different, this involves two different fields of view (figure 6) which need to be correctly overlapped with high accuracy. The relative positioning is obtained through the use of an ad-hoc calibration plate. Once spotted the points on the velocity frame, the coordinates of these points are found in object-space reference system through a minimization technique. Assumed that the thermal camera is, in good approximation, perpendicular to the acquired frame, starting from one of the points found and proceeding towards another, an aligned and larger grid which contain both frames is generated. Hence, the two measures can now be easily overlapped.

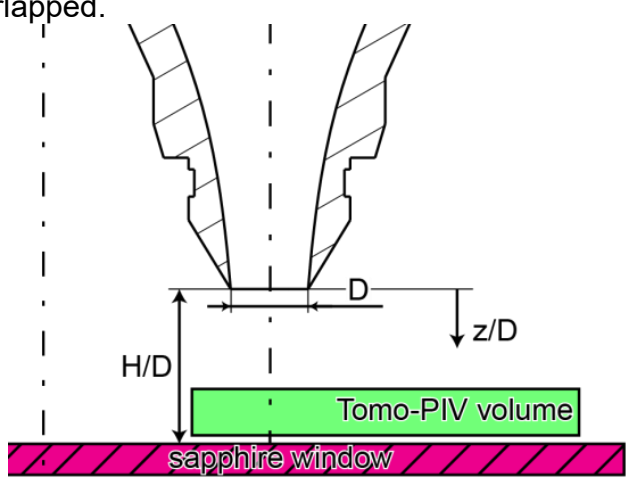

Fig. 5. Coordinate system and relative positioning of laser volume respect to the nozzle axis (view from side).

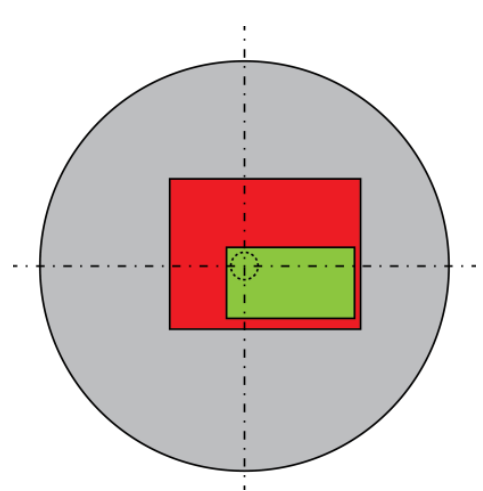

Fig. 6. Relative positioning of IR (red rectangle) and velocity (green rectangle) field of view over the circular sapphire window (view from top). The dashed circle represents the projection of the diameter.

\section{Results}

The two main actors of heat exchange and mixing are the velocity magnitude and the Turbulent Kinetic Energy (TKE). Hence, these two velocity quantities will be respectively compared to mean and standard deviation temperature maps in order to quickly associate coherent structures and patterns between the two measurements. A constant mass flow rate equal to $240 \mathrm{l} / \mathrm{h}$ is imposed resulting in a Reynolds number of 8100 . The nozzle exit is positioned above the sapphire window at $\mathrm{H} / \mathrm{D}=2$. The acquisition frequency of velocity system is $1600 \mathrm{~Hz}$. On the other side, the temperature system acquisition frequency is $400 \mathrm{~Hz}$ with IT $=1100 \mu \mathrm{s}$. It is worth to remark that InfraRed thermography measurements are relative to the wall closest sub-millimetre boundary water layer while the first plane of the Time Resolved Tomo-PIV measurements is placed $0.173 \mathrm{D}$ over the wall.

All axes reported are dimensionless and centred on jet impingement. In addition, in each image the nozzle pattern projection is reported. All velocity quantities are normalized with respect to the average bulk velocity evaluated from mass flow rate and nozzle diameter $\left(\mathrm{W}_{0}=0.84 \mathrm{~m} / \mathrm{s}\right)$. The axial velocity $w$ is always considered positive if directed from the nozzle to the wall.

The results described in [13] will be taken as reference in the following discussion.

\subsection{Circular nozzle}

The interaction of the circular jet with the impinged wall is reported in figure 7 and figure 8 . In the first image the total velocity magnitude in percentage of the average bulk velocity is placed over the mean temperature map. The mean temperature pattern is perfectly axial symmetric with respect to impingement centre. Because of the short $H / D$ distance 
the velocity profile of the jet does not have sufficient room to develop in a decaying jet. As a matter, the velocity is essentially uniform in that region [1]. Moving from the impingement centre the temperature profile experiences a plateau up to 1D and then it rises up. This behaviour is due to flow field morphology. As a matter, the mean velocity contour (in white) is axial symmetric but it seems not to perfectly imitate the temperature profile. This can be ascribed to the fact that the average of velocity quantities was evaluated over 1700 samples which correspond to an acquisition time of $1.0625 \mathrm{~s}$. In fact, moving away from the centreline the velocity profile experiences a maximum located at $0.6 \mathrm{D}$ caused by the decaying of potential core impingement and of the enforcing of the wall jet regime. From thermal quantities the integral time scale has been estimated to be equal to $35 \mathrm{~ms}$ which means that only about 30 statistically representative samples has been acquired. Hence, the sample is clearly not sufficient to obtain an accurate estimation of the mean flow field.

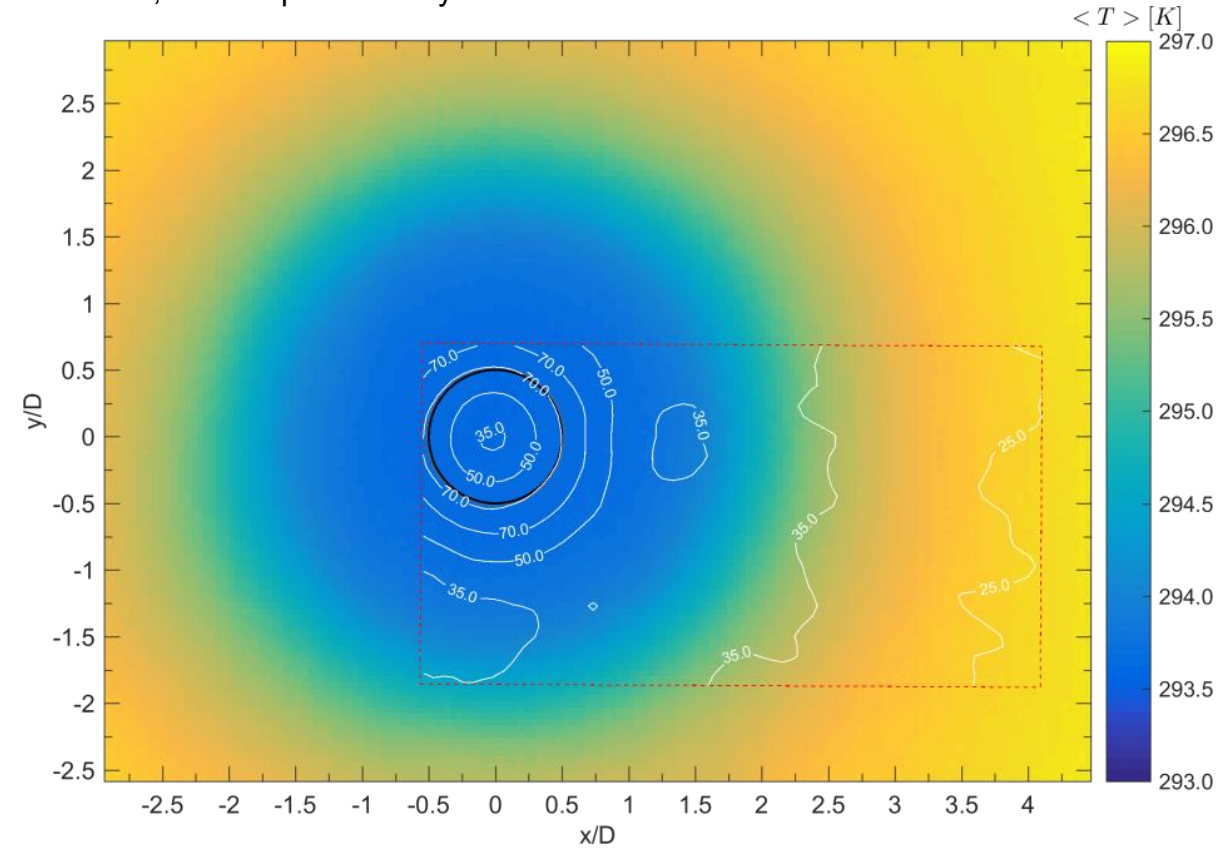

Fig. 7. (Circular case) - White contour) Normalized total velocity magnitude (in percentage); coloured map) mean temperature.

In figure 8 the TKE contour magnified of a factor $10^{3}$ is overlapped over the standard deviation temperature map. The standard deviation of temperature map is explanatory to spot the high mixing regions and the potential core region. As a matter, the region in proximity of the impingement centre shows temperature fluctuations of less than $0.05 \mathrm{~K}$. Downstream of the stagnation region the flow spreads radially and parallel to the impinged plate and develops in a semiconfined flow (wall-jet region). At the specific location of $1.75 \mathrm{D}$ there is the presence of the maximum temperature fluctuation [13]. This betrays the reattachment of a recirculation region where the cold and the hot fluid mix. Beyond that location the temperature fluctuations weakly reduce along the development and decaying of the wall jet region. From the velocity point of view the jet is characterized by a local minimum of axial velocity in the stagnation region. Then the fluctuations rapidly increase until reaches a maximum in proximity of 1D of distance from the impact point. 


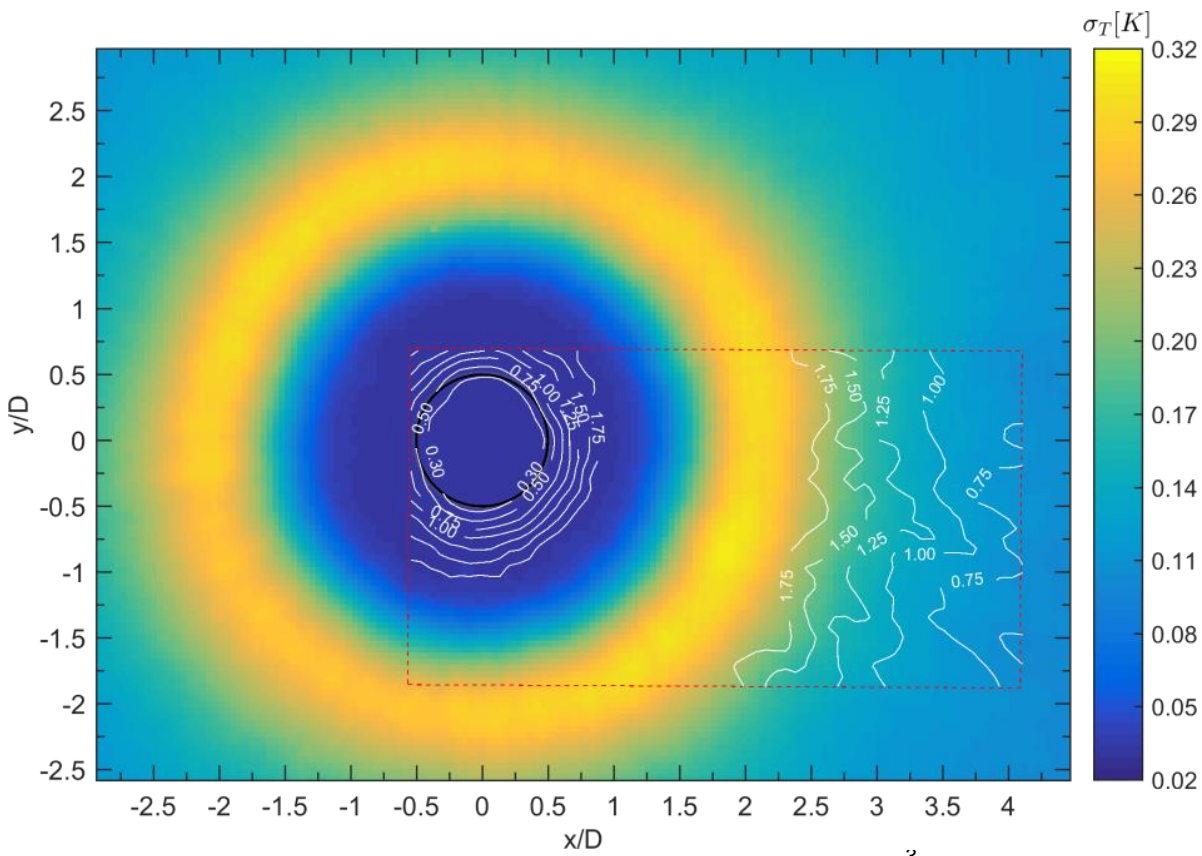

Fig. 8. (Circular nozzle) - White contour) TKE magnified of a factor $10^{3}$; coloured map) standard deviation temperature.

In figure 9 a three-dimensional representation of the circular jet case as isosurface plots of different quantities is shown. The isosurface in red color corresponds to the axial velocity surface corresponding to the value $w / W_{0}=0.53$. This can be associated with good approximation with the potential core impinging on the plate. It approaches the wall still undisturbed because of the short room available between the nozzle exit and the impinged wall. In green color the normalized TKE corresponding to the value $2.3 \cdot 10^{-3}$ is pictured. The TKE blanket spreads from the core jet with an evident ripple placed at distance of $1.75 \mathrm{D}$. As background of the $3 \mathrm{D}$ frame it is represented the normalized total velocity magnitude.

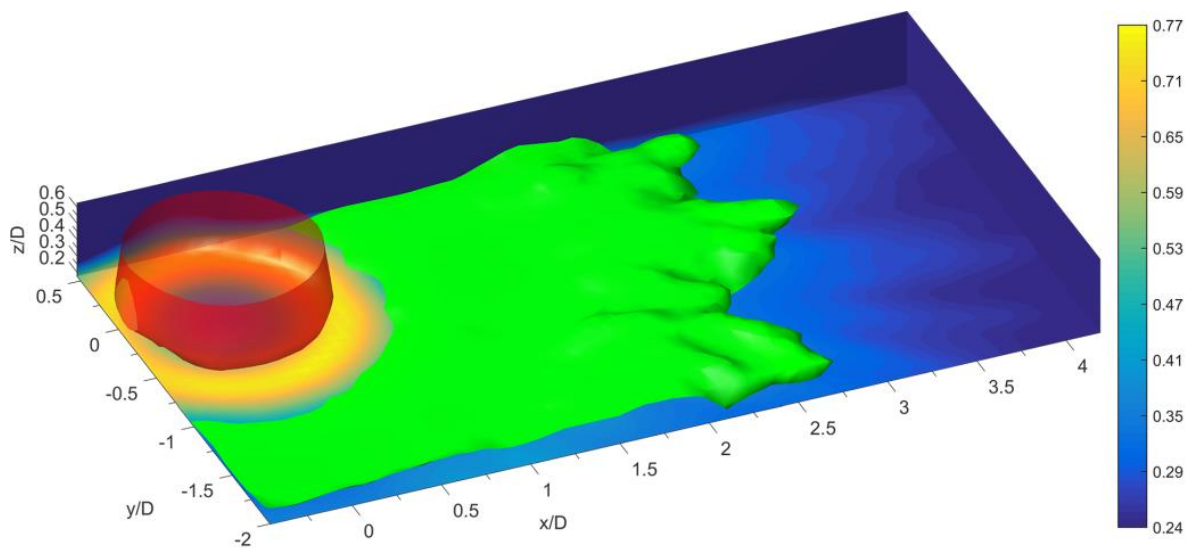

Fig. 9. (Circular nozzle) isosurface of axial velocity $w / W_{0}=0.53$ (in red), isosurface of TKE $/ W_{0}^{2}=2.3 \cdot 10^{-3}$ (in green), normalized total velocity magnitude on the background (colorbar on the right).

\subsection{Chevron nozzle}

Figure 10 and figure 11 are representative to describe the interaction of the chevron jet with the impinged wall. The first figure presents the mean temperature map overlapped by the contour of total velocity magnitude in percentage of the average bulk velocity. Similarly to the circular case, the chevron case is characterised by a minimum of axial velocity in proximity of the central region of impingement. On the other hand, this region is surrounded by a six-point-star shaped area which characterises both the temperature and velocity maps. This is due to the flow clearly issued from the nozzle custom shape. Comparing the velocity profiles the potential core of the chevron jet has a thinner cross-section with respect to the circular case. In addition, the chevron jet it is characterised by higher value of total velocity magnitude. The alternating pattern of high and low total velocity shows that the higher values of velocity are measured along the directions corresponding to the apices of the nozzle. This behaviour could be ascribed to the fact that the jet experiences 
an axis-switching like behaviour on the wall, as characteristic for cross-shaped continuous jets [13,24,25]. The star shaped morphology replicates on the plate generating preferential outflow paths along apex directions, resulting in high momentum flow along these directions. The temperature map shows the characteristic six-point-star shape which accurately resembles the nozzle exit shape similarly to what observed for the flow field.

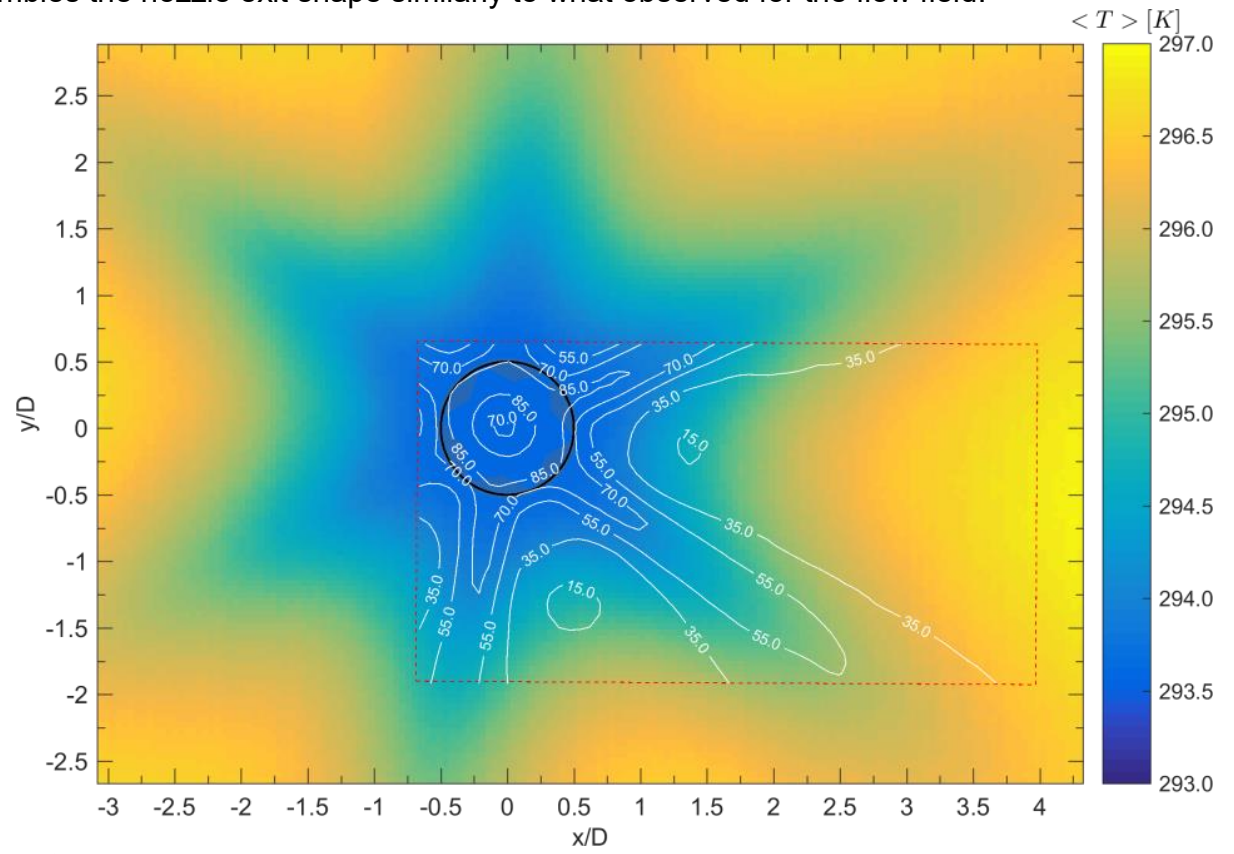

Fig. 10. (Chevron nozzle) - White contour) Normalized total velocity magnitude (in percentage); coloured map) mean temperature.

Figure 11 describes the TKE contour superimposed over the standard deviation temperature map. As first, it can be noticed that larger fluctuations values are measured along apices' directions. Here the temperature fluctuations are the smallest one. At distances larger than 3D from the centre the wall jet is completely developed and the temperature fluctuations uniform with the surrounding regions. On the other hand, the highest temperature fluctuations values are concentrated along notches' directions where happens the reorganization of the fluid impacting on the wall. In these regions, the velocity fluctuations rapidly decay moving away from the impingement centre.

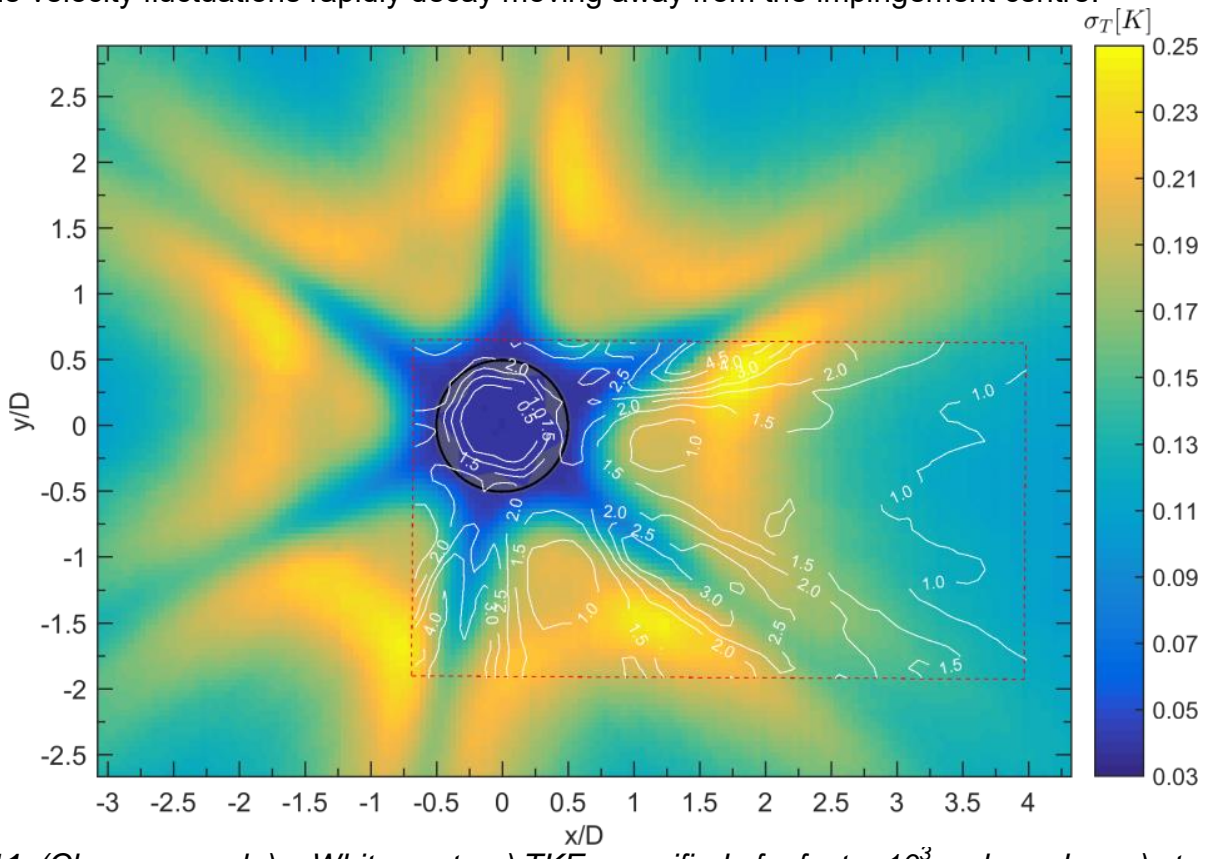

Fig. 11. (Chevron nozzle) - White contour) TKE magnified of a factor $10^{3}$; coloured map) standard deviation temperature. 
In figure 12 there is reported a three-dimensional representation of the chevron jet case. Like in the circular case the red isosurface corresponds to the value $\mathrm{w} / \mathrm{W}_{0}=0.53$ while in green color there is the normalized TKE corresponding to the value $2.3 \cdot 10^{-3}$. As background of the $3 \mathrm{D}$ frame it is depicted the normalized total velocity magnitude The remarkable difference from the circular one is the six-shaped TKE pattern departing from the core jet. The TKE distribution appears to be constant in thickness in the range of observation of the flow field. Hence, it can be deduced with good approximation that a long distance lasting heat exchange will exist along these preferential directions.

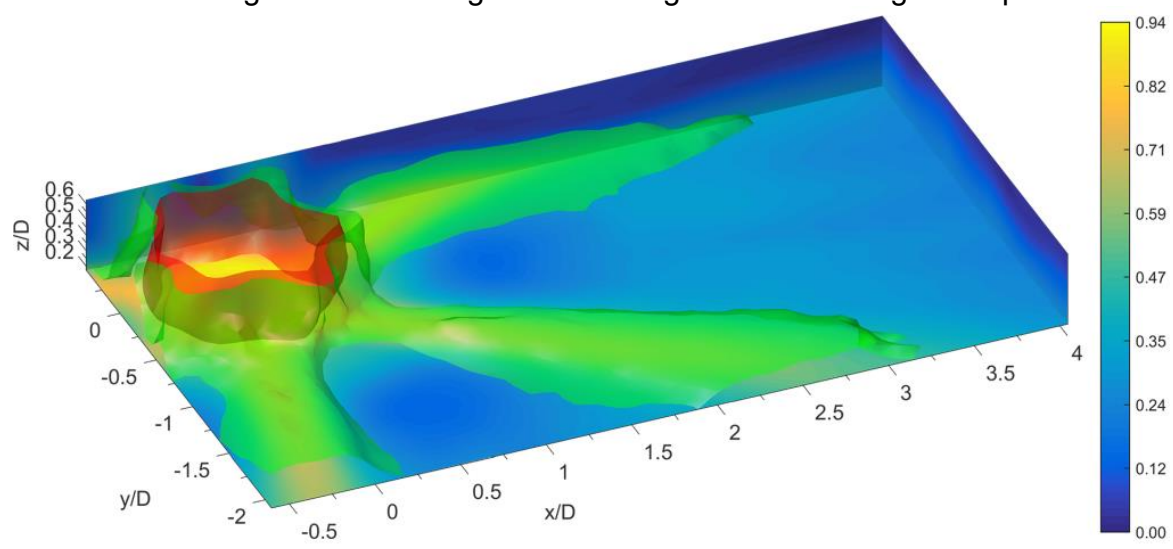

Fig. 12. (Chevron nozzle) isosurface of axial velocity $w / W_{0}=0.53$ (in red), isosurface of $T K E / W_{0}^{2}=2.3 \cdot 10^{-3}$ (in green), normalized total velocity magnitude on the background (colorbar on the right).

\section{Conclusions}

In the present work an impinging chevron jet is experimentally studied at Reynolds number equal to 8100 and compared with circular jets at the same flow regime. Flow field measurements are performed by Time Resolved Tomograhpic Particle Image Velocimetry technique. Simultaneously, at a quarter of the previous frequency of acquisition, temperature measurements are acquired through by the application of InfraRed thermography technique. Optical access for both techniques is provided by a sapphire window placed on the bottom of a tank filled with water at higher temperature $(\Delta T=5 K)$ than the impinging jet. The nozzle to plate distance is fixed at the height $H / D=2$.

According to average velocity profiles, the potential core of the chevron jet has a thinner cross-section with respect to the circular case. In addition, velocity in the impingement centre is higher in chevron case. The presence of tabs induces a star-shaped pattern on the wall, with regions of higher total velocity magnitude in correspondence to the chevron apices. The average temperature maps accurately follow the velocity pattern in both cases. The preferential directions highlighted in the chevron case can be spotted also in fluctuation velocity maps.

In the circular case, the second radial peak placed at $1.75 \mathrm{D}$ from the impingement centre is evident from temperature maps. On the other hand, the highest temperature fluctuations values are concentrated along notches' directions for the chevron nozzle. At distances larger than 3D from the impact centre the fluctuations rapidly decay resulting in a uniform distribution of temperature.

\section{REFERENCES}

[1] Carlomagno, G. M., \& laniro, A. (2014). Thermo-fluid-dynamics of submerged jets impinging at short nozzle-toplate distance: A review. Experimental thermal and fluid science, 58, 15-35.

[2] Jambunathan, K., Lai, E., Moss, M. A., \& Button, B. L. (1992). A review of heat transfer data for single circular jet impingement. International journal of heat and fluid flow, 13(2), 106-115.

[3] laniro, A., \& Cardone, G. (2012). Heat transfer rate and uniformity in multichannel swirling impinging jets. Applied Thermal Engineering, 49, 89-98.

[4] Gardon, R., \& Akfirat, J. C. (1965). The role of turbulence in determining the heat-transfer characteristics of impinging jets. International journal of heat and mass transfer, 8(10), 1261-1272.

[5] Warhaft, Z. (2000). Passive scalars in turbulent flows. Annual Review of Fluid Mechanics, 32(1), 203-240.

[6] Astarita, T., \& Carlomagno, G. M. (2012). Infrared thermography for thermo-fluid-dynamics. Springer Science \& Business Media.

[7] Westerweel, J., Elsinga, G. E., \& Adrian, R. J. (2013). Particle image velocimetry for complex and turbulent flows. Annual Review of Fluid Mechanics, 45, 409-436.

[8] Kolmogorov, A. N. (1991). The local structure of turbulence in incompressible viscous fluid for very large Reynolds numbers. Proc. R. Soc. Lond. A, 434(1890), 9-13.

[9] Gutmark, E. J., \& Grinstein, F. F. (1999). Flow control with noncircular jets. Annual review of fluid mechanics, 31(1), 239-272.

[10] Bridges, J., \& Brown, C. (2004, May). Parametric testing of chevrons on single flow hot jets. In 10th AIAA/CEAS Aeroacoustics Conference (p. 2824). 
[11] Zaman, K. B. M. Q., Bridges, J. E., \& Huff, D. L. (2011). Evolution from 'tabs' to 'chevron technology'-a review. International Journal of Aeroacoustics, 10(5-6), 685-709.

[12] Reeder, M. F., \& Samimy, M. (1996). The evolution of a jet with vortex-generating tabs: real-time visualization and quantitative measurements. Journal of Fluid Mechanics, 311, 73-118.

[13] Violato, D., laniro, A., Cardone, G., \& Scarano, F. (2012). Three-dimensional vortex dynamics and convective heat transfer in circular and chevron impinging jets. International Journal of Heat and Fluid Flow, 37, 22-36.

[14] Violato, D., \& Scarano, F. (2011). Three-dimensional evolution of flow structures in transitional circular and chevron jets. Physics of Fluids, 23(12), 124104.

[15] Prahl S., Optical absorption of water compendium https://omlc.org/spectra/water/abs/index.html

[16] Thorlabs Inc. Total Transmission of $5 \mathrm{~mm}$ Thick, Uncoated Sapphire Window https://www.thorlabs.com/images/Tablmages/WG31050_Raw_Data.xlsx

[17] Wieneke, B. (2008). Volume self-calibration for 3D particle image velocimetry. Experiments in fluids, 45(4), 549556.

[18] Elsinga, G. E., Scarano, F., Wieneke, B., \& van Oudheusden, B. W. (2006). Tomographic particle image velocimetry. Experiments in fluids, 41(6), 933-947.

[19] Wieneke, B. (2012). Iterative reconstruction of volumetric particle distribution. Measurement Science and Technology, 24(2), 024008.

[20] Schanz, D., Gesemann, S., \& Schröder, A. (2016). Shake-The-Box: Lagrangian particle tracking at high particle image densities. Experiments in fluids, 57(5), 70.

[21] Discetti, S., \& Astarita, T. (2010). Acceleration of Tomo-PIV by multigrid reconstruction schemes. In 15th International Symposium on Applications of Laser Techniques for Fluid Mechanics.

[22] Discetti, S., \& Astarita, T. (2012). Fast 3D PIV with direct sparse cross-correlations. Experiments in fluids, 53(5), $1437-1451$

[23] Viskanta, R. (1993). Heat transfer to impinging isothermal gas and flame jets. Experimental thermal and fluid science, 6(2), 111-134.

[24] Rau, M. J., Dede, E. M., \& Garimella, S. V. (2014). Local single-and two-phase heat transfer from an impinging cross-shaped jet. International Journal of Heat and Mass Transfer, 79, 432-436.

[25] Chen, N., \& Yu, H. (2014). Mechanism of axis switching in low aspect-ratio rectangular jets. Computers \& Mathematics with Applications, 67(2), 437-444. 